\title{
Screening of Lactococcal Adhesion Genes and Two Pneumococcal Genes as Genetic Determinants of Virulence in Lactococcus garvieae Strains
}

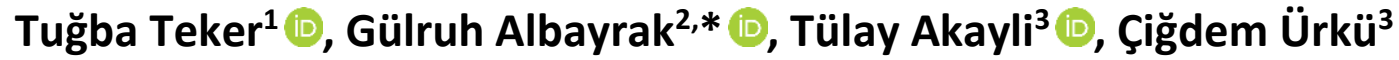

\author{
${ }^{1}$ Istanbul University, Institute of Graduate Studies in Sciences, Programme of Molecular Biotechnology and Genetics, \\ Istanbul, Turkey \\ ${ }^{2}$ Istanbul University, Faculty of Science, Department of Molecular Biology and Genetics, Istanbul, Turkey. \\ ${ }^{3}$ Istanbul University, Faculty of Aquatic Sciences, Department of Fish Diseases, Istanbul, Turkey.
}

\section{Article History}

Received 10 June 2020

Accepted 16 July 2020

First Online 24 July 2020

\section{Corresponding Author \\ Tel.: +902124555700 \\ E-mail: gulruh@istanbul.edu.tr}

\section{Keywords}

Lactococcosis

Polymerase Chain Reaction

Virulence determinant

Adhesin

\begin{abstract}
Nine lactococcal adhesin and two pneumococcal virulence genes were characterized in twenty Lactococcus garvieae strains obtained from rainbow trout, and also one human isolate by PCR. Findings showed that all strains carried adhPsaA (522 bp), LPXTG-1 (947 bp), adhCl (490 bp), and purB (864 bp) while some of the strains had adhPav (1048 bp), LPXTG-2 (767 bp), LPXTG-3 (231 bp), adhCII (732 bp), adh (398 bp) and SP_0121 (966 bp). High nucleotide homologies (85-99\%) of adhCl, adhCll, adh, adhPav, adhPsaA, LPXTG-1, LPXTG-3 involved in bacterial adhesion were determined between $L$. garvieae strains and reference Lg2. Genes containing polymorphisms among strains were not considered to be directly involved in bacterial pathogenesis. The amplification of LPXTG-3 only in fish isolates showed that it might be responsible for coding the host-specific virulence factor. However, undetermined amplicons demonstrated that LPXTG-4 could not be used as a host-specific gene marker. Pneumococcal purB and $S P_{-} 0121$ have been experimentally detected in L. garvieae genome for the first time. Consequently, both purB and SP_0121 can be used as a virulence marker for $L$. garvieae. Findings provided valuable knowledge about $L$. garvieae pathogenesis and, will contribute to identify the appropriate genomic targets to develop new therapeutics against the lactococcosis.
\end{abstract}

\section{Introduction}

The etiologic agent of lactococcosis is Lactococcus garvieae which is one of the most significant pathogens for marine and freshwater aquaculture. Emerging disease affects many fish species. L. garvieae usually causes endemic infections, resulting in significant economic losses in the aquaculture industry (Vendrell et al., 2006; Wang et al., 2007). In addition, this pathogen causes disease in human who consumes raw fish (Wang et al. 2007; Tandel et al., 2017).

The ability of an organism to cause disease is called virulence (Casadevall \& Pirofski, 1999). Bacterial adhesion, defined as the attachment of bacteria to various surfaces, is effective in virulence by facilitating the invasion and colonization of host tissues (Chhatwal, 2002). Adhesion-related cell surface proteins are identified as four main types: (1) cytoplasmic membrane proteins, (2) lipoproteins which are covalently attached to membrane lipids (3) proteins containing carboxyl terminal LPxTG-like motif (4) proteins with specific domains that recognize certain cell wall components (Desvaux et al., 2006; Kleerebezem et al. 2010).

A number of adhesion genes possibly involved in virulence factors were identified in L. garvieae Lg2 (Miyauchi et al., 2012). Products of adhesin gene cluster 1, $-2(a d h \mathrm{Cl}, a d h \mathrm{Cl})$, adhesin gene $(a d h)$, adhesin Pav (adhPav), adhesin PsaA (adhPsaA), LPxTG containing 
surface protein 1, - 2, -3, -4 genes (LPXTG-1, LPXTG-2, LPXTG-3, LPXTG-4) in L. garvieae, have high amino acid sequence identity between orthologs. Therefore, these genes have been screened as virulence determinants in L. garvieae (Morita et al., 2011; Miyauchi et al., 2012; Ture \& Altinok, 2016).

Discovering virulence factors is one of the most important steps to develop novel drugs and new vaccines for the treatment and prevention of bacterial pathogens. Determination of these factors not only contributes to the knowledge about the pathogenesis mechanism of bacteria but also can be used as new targets for therapeutics. Pathosystem resource integration center (PATRIC) is a bioinformatic resource center which supports relevant basic and applied biomedical researches about bacterial infectious diseases (Gillespie et al., 2011; Wattam et al., 2014). PATRIC is an integrated system associated with a wide variety of data banks. Also, a wide variety of data, such as genomic, transcriptomics, protein-protein interactions, protein 3D structure can be demonstrated through this bioinformatic tool. Putative virulence factors, which are responsible for the virulence of phylogenetically close species, but not in L. garvieae, can be predicted by the PATRIC web interface together with their nucleotide sequences.

Virulence factors responsible for adhesion in different pathogenic bacteria were revealed by annotated and validated similarity searches (Morita et al., 2011). The main goal of the current study is the characterization of these adhesion genes in L. garvieae strains which were recovered from infected fish tissues and one isolate obtained from a human sample, together with a reference strain. It was also aimed to determine the pneumococcal virulence genes as new candidate virulence determinants in L. garvieae by using bioinformatic tools and PCR.

\section{Materials and Methods}

\section{Identification of Bacterial Strains and Growth Conditions}

Twenty-one $L$. garvieae strains, which were grown on Todd Hewitt Broth (THB) medium and incubated aerobically at $25^{\circ} \mathrm{C}$ for $24 \mathrm{~h} .$, were listed in Table 1 . Among them 19 strains were isolated from different organs (heart, liver, kidney, blood, spleen) of diseased rainbow trout (Oncorhynchus mykiss), one strain from infected human (FMB-H). And the type strain L. garvieae ATCC 43921 was obtained from American Type Culture Collection (FMB-R). Molecular confirmation by PCR of $16 \mathrm{~S}$ rRNA gene region and phenotypic characterization of all strains using rapid ID 32 Strep (Biomérieux) were previously performed by Teker et al. (2019).

\section{Investigation of Virulence Genes through PATRIC}

PATRIC web page (https://www.patricbrc.org/5) was used to determine potential virulence genes for $L$. garvieae. "Lactococcus garvieae" was entered as data to the "Specialty Genes" tab. This tab opened up a filter where it can be filtered on property, source, or evidence. "Victors" was selected as the source, and virulence factors, which were identified in the phylogenetically close species to L. garvieae, were monitored. Nucleotide sequences of Pneumococcal genes, encoding virulence factors, were obtained from National Center for Biotechnology Information (NCBI). The presence of them was analyzed in $L$. garvieae genome by CLUSTALW.

\section{PCR Amplification and Sequence Analysis}

Oligonucleotides were designed for the amplification of seven virulence genes (adhCl, adhCll, adh, adhPav, adhPsaA, LPXTG-1, LPXTG-4) and also two potential virulence factor genes (SP_0121 and purB). The primer pairs p0842, p0843, p0196, p1330, p1533, p1005, p1672, Psp, PPur were designed by "Primer3" program. Also, primer pairs (LP2, LP3, LP4), which were designed by Ture and Altinok (2016), were used to amplify three LPXTG genes (LPXTG-2, -3, -4) (Table 2).

The genomic DNAs (gDNAs) of all strains were extracted by using High Pure PCR Template Preparation Kit (Roche) according to the manufacturer's instructions. Quality and quantity of gDNAs and their integrity and purity were controlled through spectrophotometrically and by the gel electrophoresis, respectively (Maniatis, Fritch, \& Sambrook, 2003).

Table 1. Origin of L. garvieae strains

\begin{tabular}{|c|c|c|c|}
\hline Strain & Location of Source & Strain & Location of Source \\
\hline FMB-F1 & Fethiye/Turkey & FMB-F12 & Fethiye/Turkey \\
\hline FMB-F2 & Fethiye/Turkey & FMB-F13 & Fethiye/Turkey \\
\hline FMB-F3 & Fethiye/Turkey & FMB-F14 & Fethiye/Turkey * \\
\hline FMB-F4 & Fethiye/Turkey & FMB-F15 & Fethiye/Turkey * \\
\hline FMB-F5 & Fethiye/Turkey & FMB-F16 & Fethiye/Turkey * \\
\hline FMB-F6 & Fethiye/Turkey & FMB-BL1 & Bafra Lake/Turkey \\
\hline FMB-F7 & Fethiye/Turkey & FMB-BL2 & Bafra Lake/Turkey \\
\hline FMB-F8 & Fethiye/Turkey & FMB-F17 & Fethiye/Turkey ** \\
\hline FMB-F9 & Fethiye/Turkey & FMB-H & Human isolate/Turkey \\
\hline FMB-F10 & Fethiye/Turkey & ATCC 43921 (FMB-R) & Culture Collection/United Kingdom \\
\hline FMB-F11 & Fethiye/Turkey & & Culture Collection/United Kıngdom \\
\hline
\end{tabular}

\footnotetext{
*: obtained from different hatchery, ${ }^{* *}$ : first isolate reported from Turkey
} 
Amplification of the genes was performed in a 25 $\mu \mathrm{l}$ reaction mixture containing; $25 \mathrm{ng}$ bacterial gDNA, $1 \mathrm{X}$ PCR buffer, $2.5 \mathrm{mM} \mathrm{MgCl} 2,0.4 \mathrm{mM}$ dNTP mix, 15 pmol of primer pairs, and $1 \mathrm{U}$ of Taq DNA polymerase (Thermo). Pre-denaturation of gDNAs was carried out at 95 oC for $2 \mathrm{~min}$. The PCR was achieved following conditions consisting of 28 cycles: at $95^{\circ} \mathrm{C}$ for 45 seconds of denaturation, at $52-60^{\circ} \mathrm{C}$ (Table 2) for 45 seconds of annealing, and finally at $72^{\circ} \mathrm{C}$ for 1.5 minutes of extension. The final extension was completed at $72{ }^{\circ} \mathrm{C}$ for 7 minutes. Instead of template gDNA, double distilled water was used in the sample of negative control. All of the reactions were repeated three times to ensure reliability.

PCR was conducted in volume of $50 \mu \mathrm{l}$ for DNA sequencing and amplicons were purified with the Agarose Gel DNA Extraction Kit (Roche) according to the manufacturer's instructions. Purified PCR products were sequenced based on Sanger method by RefGen. Comparison of nucleotide sequence homologies among L. garvieae strains and reference $L$. garvieae strain Lg2 was performed by using ClustalW (http://www.genome.jp/tools-bin/clustalw) online web tool.

\section{Results}

Analysis of Lactococcal Adhesin Genes

A 522 bp fragment of the adhPsaA gene (Figure 1a), $947 \mathrm{bp}$ of the LPXTG-1 gene (Figure $1 \mathrm{~b}$ ) and $490 \mathrm{bp}$ of the $a d h \mathrm{Cl}$ (Figure 1c) were amplified from the gDNAs of all $L$. garvieae strains. The adhCll gene with the size of 732 bp (Figure 1d), adhPav gene fragment with 1048 bp (Figure 1e) and adh product with $398 \mathrm{bp}$ (Figure 1f) could not be produced from FMB-F13, FMB-BL1, FMBBL2, FMB-F17, FMB-R, FMB-H strains. The LPXTG-2 gene product with the size of $767 \mathrm{bp}$ was amplified from six strains (FMB-F1, FMB-F15, FMB-F16, FMB-BL2, FMBF17, FMB-R) (Figure 1g). The LPXTG-3 gene fragment (231 bp) was yielded from all bacterial strains except FMB-H (Figure 1h). Also, amplification profiles belonging to these genes were given in Table 3.

The expected 928 and 1021 bp length partial region of LPXTG-4 gene fragment was not amplified through PCR by using LP4 primer (chosen from literature) and p1672 primer (designed for the current study). However, p1672 primer pairs amplified a single unexpected fragment with $1500 \mathrm{bp}$ in 10 strains and two

Table 2. List of primer pairs, which were used for the amplification of virulence genes, localized in different locus; their annealing and product sizes.

\begin{tabular}{|c|c|c|c|c|c|}
\hline Primer & Primer sequence (5'-3') & Target gene & Locus & АT $\left({ }^{\circ} \mathrm{C}\right)$ & PS (bp) \\
\hline p1533 & $\begin{array}{l}\text { fCGGGAAGGACCATGTTGATG } \\
\text { rAGTTGGGCTGGTGTACCTTG }\end{array}$ & adhPsaA & LCGL 1533 & 59 & 552 \\
\hline p1005 & $\begin{array}{l}\text { 'TACGCATCCGCAAGGAGC } \\
\text { 'CTGCAACATTACCACGCACT }\end{array}$ & LPXTG-1 & LCGL 1005 & 57 & 947 \\
\hline p0842 & $\begin{array}{l}\text { fCAGCTACTACAGGGTTCGC } \\
\text { rGCATCATCAGCTGCCAAGTTG }\end{array}$ & adhCl & LCGL 0842 & 58 & 490 \\
\hline p0843 & $\begin{array}{l}\text { 'TGATTACACACCCAGCTCCA } \\
\text { rCTTTTTCCTAGCCCGAAAGC }\end{array}$ & adhCll & LCGL 0843 & 57 & 732 \\
\hline p1330 & $\begin{array}{l}\text { fGACACAGACCTTGCAGTCCA } \\
\text { rGATGACGGACTCATCAGGTG }\end{array}$ & adhPav & LCGL 1330 & 59 & 1048 \\
\hline p0196 & $\begin{array}{l}\text { fGTTGTCACAGAACCGGGGC } \\
\text { 'TCTCCTGCGTTGACATGGAC }\end{array}$ & $a d h$ & LCGL 0196 & 60 & 398 \\
\hline LP2* & $\begin{array}{l}\text { fGCCAGTGAGAGAACCGTTGA } \\
\text { rCAGGTTCAAGTGCAACTGCC }\end{array}$ & LPXTG-2 & LCGL 1410 & 57 & 767 \\
\hline LP3* & $\begin{array}{l}\text { 'TTAAGCACAACGGCAACAGC } \\
\text { 'CACGCGAAATGATGGTGCAT }\end{array}$ & LPXTG-3 & LCGL 1585 & 55 & 231 \\
\hline p1672 & $\begin{array}{l}\text { 'TAAGCCGTGTTGGTCTGAAG } \\
\text { 'TCCGTTTACTGACAAAGCCG }\end{array}$ & LPXTG-4 & LCGL 1672 & 57 & 1021 \\
\hline LP4* & $\begin{array}{l}\text { fGGGAGCACCGGATTCACTTT } \\
\text { 'ACAAAGCCGCAGACCTTACA }\end{array}$ & LPXTG-4 & LCGL 1672 & 52 & 928 \\
\hline Ppur & $\begin{array}{l}\text { fCTGGTCGGACGAAAACAAAT } \\
\text { rCGTTCATGCCACAAAACAAC }\end{array}$ & purB & LCGL 0565 & 55 & 864 \\
\hline Psp & $\begin{array}{l}\text { fACATGCGGTACTCACCATGA } \\
\text { rACGGACACGAGGACCACATC }\end{array}$ & $S P \_0121$ & SP 0121 & 60 & 966 \\
\hline
\end{tabular}

${ }^{f}$ : forward primer, ${ }^{r}$ : reverse primer, AT: annealing temperature, PS: product size 
Table 3. The amplification profiles belonging to adhCII, adhPav, adh, LPXTG-2, LPXTG-3, SP_0121 genes of L. garvieae strains.

\begin{tabular}{|c|c|c|c|c|c|c|}
\hline \multirow{2}{*}{ Strain } & \multicolumn{6}{|c|}{ Gene } \\
\hline & adhClI & adhPav & $a d h$ & LPXTG-2 & LPXTG-3 & SP_0121 \\
\hline FMB-F1 & + & + & + & + & + & + \\
\hline FMB-F2 & + & + & + & - & + & + \\
\hline FMB-F3 & + & + & + & - & + & + \\
\hline FMB-F4 & + & + & + & - & + & + \\
\hline FMB-F5 & + & + & + & - & + & + \\
\hline FMB-F6 & + & + & + & - & + & + \\
\hline FMB-F7 & + & + & + & - & + & + \\
\hline FMB-F8 & + & + & + & - & + & + \\
\hline FMB-F9 & + & + & + & - & + & + \\
\hline FMB-F10 & + & + & + & - & + & + \\
\hline FMB-F11 & + & + & + & - & + & + \\
\hline FMB-F12 & + & + & + & - & + & + \\
\hline FMB-F13 & - & - & - & - & + & - \\
\hline FMB-F14 & + & + & + & - & + & + \\
\hline FMB-F15 & + & + & + & + & + & + \\
\hline FMB-F16 & + & + & + & + & + & + \\
\hline FMB-BL1 & - & - & - & - & + & + \\
\hline FMB-BL2 & - & - & - & + & + & - \\
\hline FMB-F17 & - & - & - & + & + & + \\
\hline FMB-R & - & - & - & + & + & + \\
\hline FMB-H & - & - & - & - & - & + \\
\hline
\end{tabular}

+: presence of the amplification product; -: absence of the amplification product

amplicons (approximately 250 and 500 bps) in three strains.

It was found that the nucleotide homology of adhPsaA, LPXTG-1, adhCl, adhCII, adhPav, adh, LPXTG-3 genes between $L$. garvieae strains and $L$ 2 was varied from $85 \%$ to $99 \%$. FMB-F11 and FMB-F12 had the highest sequence similarities with reference strain Lg2. The nucleotide sequences of adhPsaA, LPxTG-1, adhCl, adhCll, adh, LPXTG-3 genes belonging to FMB-F12 were registered in the GenBank under the accession numbers MK177896, MK177895, MK177893, MK177894, MK177892, MK177897, respectively.

\section{Bioinformatic and Molecular Analysis of Pneumococcal Genes in L. Garvieae}

PATRIC data displayed that, there was $99 \%$ amino acid sequence homology of virulence factors, encoded by purB gene in S. pneumoniae D39 strain and $L$. garvieae TRF1 strain (Lau et al., 2001). In addition, amino acid similarity of virulence factor, encoded by SP_0121 gene, was $84 \%$ in S. pneumoniae TIGR4 and $L$. garvieae Lg2. It was thought that both pneumococcal purB and SP_0121 genes could also be screened as virulence determinants in L. garvieae according to these BLASTP values.

The size of amplicons of pneumococcal purB and SP_0121 genes were 864 bp (Figure 2a) and 966 bp (Figure $2 b$ ) length, in $L$. garvieae strains, respectively. It was revealed that all strains carried the purB gene, whereas the SP_0121 gene fragment was not produced from FMB-F13 and FMB-BL2 (Table 3). PCR amplifications have supported the outcomes of bioinformatic analysis.
BlastN analysis of the pneumococcal genes showed that nucleotide sequence homology among strains and Lg2 was varied from 93-99\% for purB and \%95-97 for SP_0121. Sequence data of purB gene in FMB-F12 strain, obtained from the current study, was deposited the GeneBank under accession number MK227456.

\section{Discussion}

Adhesins are cell surface proteins synthesized by bacteria and play a role in host-pathogen interactions. Nine genes (adhPsaA, adhPav, LPxTG-1, LPXTG-2, LPXTG3, LPXTG-4, adhCl, adhCll ve $a d h$ ) responsible for the adhesion were screened in totally 21 L. garvieae strains, in the current study. Among them, it was found that adhPsaA, adhCl and LPxTG-1 genes were carried by all strains. However, strains were polymorphic according to the presence of adhPav, LPxTG-2, LPxTG-3, LPxTG-4, $a d h C l l$ ve $a d h$ genes in their genomes. Determination of the presence of $a d h \mathrm{PsaA}$ and $a d h \mathrm{Cl}$ genes in all strains, and the detection of adh gene polymorphisms among the stains, were similar to findings of Ture and Altinok (2016). However, different results in terms of adhPav, LPXTG-1, LPXTG-2, LPXTG-4 and adhCll genes have been obtained from this study. It was concluded that the genes amplified from all $L$. garvieae strains' genomes may be required for virulence of the bacteria, but not in the polymorphics.

Ture and Altinok (2016) reported that adhPav, LPXTG-2 and adhCII genes have been carried by all $L$. garvieae strains. But, in this study, the amplification of adhPav and adhCll genes (1048 bp and 732 bp, respectively) could not be performed from FMB-F13, FMB-BL1, FMB-BL2, FMB-F17, FMB-R, FMB-H strains. 


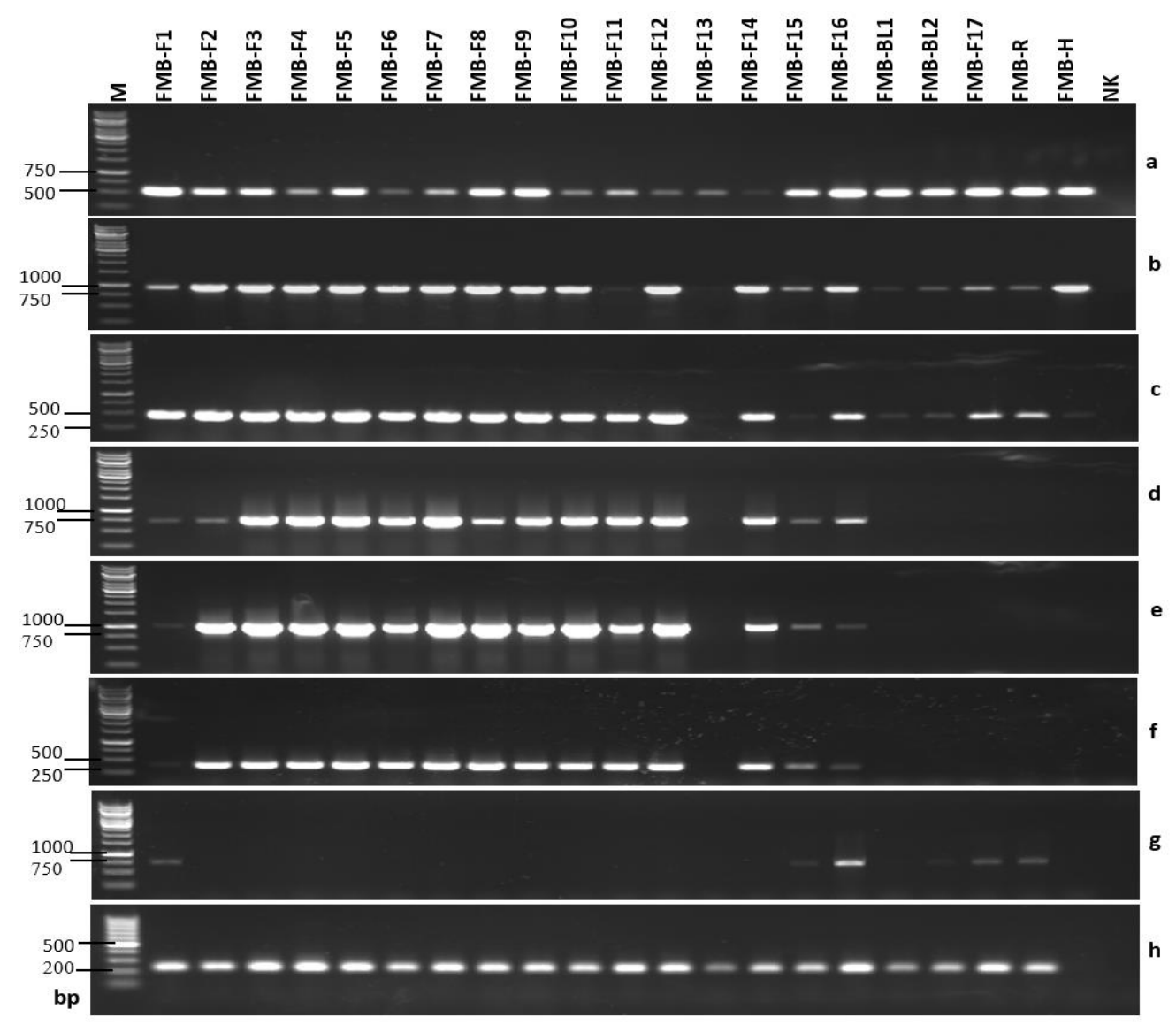

Figure 1. Analysis of adhPsaA amplicons with $522 \mathrm{bp}(\mathrm{a})$, LPxTG-1 gene products with $947 \mathrm{bp}$ (b), $490 \mathrm{bp}$ adhCl (c), $732 \mathrm{bp}$ adhCll (d), 1.048 bp adhPav (e), 398 bp adh (f), 767 bp LPXTG-2 (g), 231 bp LPXTG-3 gene fragments (h). M: 1 kb DNA ladder (Thermo Scientific) (a-g), M: 100 bp DNA ladder (Thermo Scientific) (h), N: Negative control.

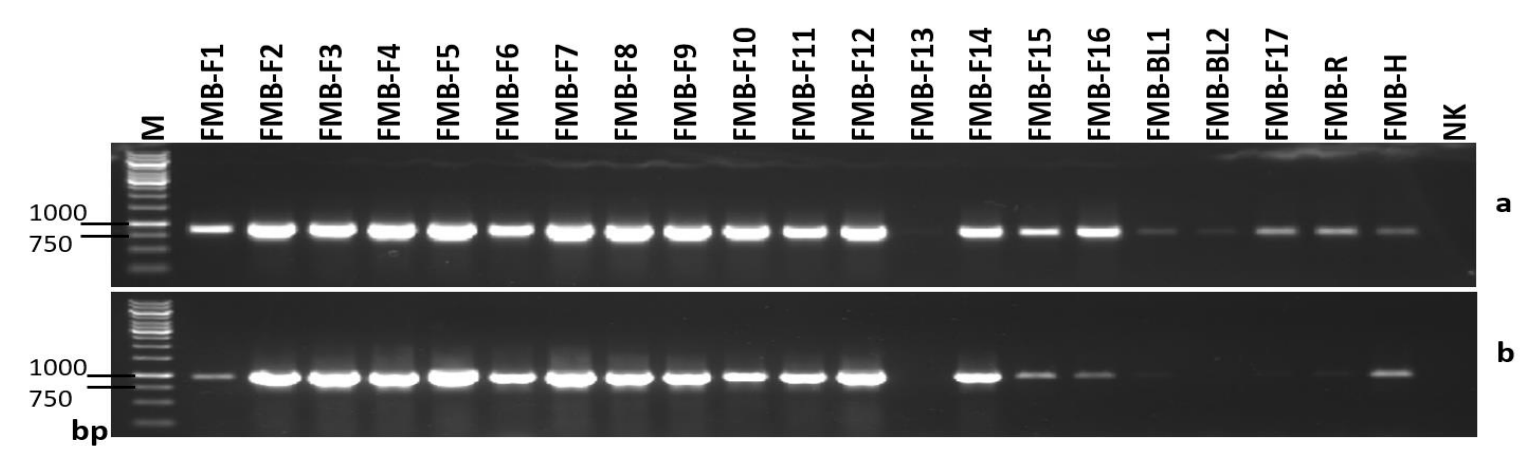

Figure 2. PCR products of purB with $864 \mathrm{bp}(\mathrm{a})$, and $966 \mathrm{bp}$ gene fragments of SP_0121 (b) in 21 L. garvieae strains. M: 1 kb DNA ladder (Thermo Scientific); N: Negative control.

Moreover, LPXTG-2 amplicons (767 bp) were produced from only 6 strains' genomes (FMB-F1, FMB-F15, FMBF16, FMB-BL2, FMB-F17, FMB-R). Therefore, it was revealed that the virulence factors associated with these genes could carry variation within the $L$. garvieae genomes.

Fragments (947 bp) which were associated with LPXTG-1 gene were identified in reference strain ATCC 43921, in the current study. However, LPXTG-1 was not produced in the ATCC 43921 by Ture and Altinok (2016). As a result, the reference strain exhibited different amplification profile in this study. Similar data to these controversial findings were also reported by Morita et al. (2011). They stated that reference strain ATCC 49156 lost its capsule gene cluster during sub culturing and undergone phenotypic changes. The sub culturing could cause the observation of different genotypic and phenotypic profiles even in the common bacterial strain. Therefore, it has great importance to the screening and identification of virulence factor genes of disease agents in the improvement of new strategies to combat the disease. 
Proteins with the LPxTG motif contain aminoterminal signal peptide, carboxy-terminal hydrophobic region and a charged tail. Although they have different functions, these proteins have been shown to carry the motif as consensus (Chhatwal, 2002). Gibello et al. (2016) reported that LPxTG proteins of L. garvieae effective in adhesion and entry process to nonphagocytic cells of rainbow trout. LPXTG-1, -2, -3, -4 genes are responsible for the coding of surface proteins which contain LPxTG motif in L. garvieae (Morita et al., 2011; Miyauchi et al., 2012). Bacterial strains isolated from different host, such as human and fish, have unique the adhesion genes. It has been reported that the LPXTG-3 and LPXTG-4 genes, which were identified in fish samples, were not present in L. garvieae strains, isolated from human (Miyauchi et al., 2012; Gibello et al., 2016). It was showed that infected human isolate (FMB-H) did not carry the LPXTG-3 gene, whereas the all $L$. garvieae, which were disease agent in fish, possessed the $L P X T G-3$, in the present study. Consequently, the results confirmed that $L P X T G-3$ is responsible for encoding host specific virulence factor.

The amplification of LPXTG-4 gene, in some strains isolated from fish pathogens were reported by Ture and Altinok (2016). In the current study, two different primer pairs (LP4 and p1672) were used for the production of the $L P X T G-4$ gene region. Primer pair, which were taken from literature (Ture \& Altinok, 2016) failed in the amplification of the expected PCR products (Table 2), whereas fragments with different sizes were obtained by using specific primers ( $p 1672$ ) designed for the first time. The detection of two different fragments of about 500 bp and 250 bp in three strains by using P1672 primers, suggested that LPXTG-4 could be found with variation in the population or the potential pseudogene of its gene could be carried in the L. garvieae genome. This finding has also great importance as it suggests that the LPXTG-4 gene may not be necessary for bacterial adhesion to the host organism. In contrast to the literature, our study revealed that LPXTG-4 might not be used as a host specific gene marker. Since some genes responsible for basic metabolic pathways are essential for bacterial growth and survival in host during infection, they might be utilized as virulence indicator (Lau et al., 2001).

The purB and SP_0121 genes are functional in the pathogenesis of $S$. pneumoniae D39 and TIGR4 strains, respectively (Lau et al., 2001; Hava \& Camilli, 2002). Their functions were detected by signature tagged mutagenesis. While purB gene encodes the adenosyl succinate lyase enzyme involved in purine biosynthesis, $S P \_0121$ codes the metallo- $\beta$-lactalbumin protein family catalyzing the hydrolysis of metallo-beta-lactam antibiotics. In silico analysis of homologous nucleotide sequences indicated that both two pneumococcal genes may be responsible for virulence in $L$. garvieae. Although it has been detected that sequences related to purB is located in LCGL 0565 locus in L. garvieae (Morita et al.,
2011), there is no data in the literature about the relationship of this gene with virulence.

Experimental demonstration of two pneumococcal genes in L. garvieae genome was conducted for the first time. Since purB (864 bp) gene was carried in all strains in this study, it can be nominated as target gene for virulence studies. In addition, the presence of SP_0121 gene was investigated in L. garvieae genome. SP_0121 gene fragment (966 bp) was amplified by all strains except FMB-F13 and FMB-BL-2. The fact that almost all strains possessed the SP_0121 partial gene fragment was thought that this pneumococcal gene can be used as a virulence marker in $L$. garvieae strains.

The results of multiple alignment analysis revealed that nucleotide sequence of adhPsaA, LPXTG-1, adhCl, adhCII, adhPav, adh, LPXTG-3 genes are highly consistent with the partial nucleotide sequences of the Lg2 NC_017490 reference genome in GenBank. Additionally, high nucleotide homology of purB and moderate similarity of SP_0121 was detected between L. garvieae strains, which were included in the current study, and reference genome Lg2. Consequently, the existence of pneumococcal genes was verified in lactococcal genome. At the same time, pneumococcal virulence gene determinants should be monitored during host-pathogen interaction process.

The pathogenicity, observed as a consequence of the host-pathogen interaction, is ability of the pathogen to cause disease (Casadevall \& Pirofski, 2000). Preventing health problems and even deaths caused by bacterial infectious diseases can be achieved by elucidation of the genetic mechanism of virulence. Although pathogenicity occurs as a consequence of the interaction of various virulence factors (Zecconi et al., 2006), determination of the effective factors in virulence, and of the genes encoding these factors enable the identification of the basic characteristics in pathogen virulence. For this reason, it is important to determine the prevalence of virulence genes in $L$. garvieae.

\section{Conclusion}

The lactococcal adhesion genes were characterized in L. garvieae genome. Also, determination of two pneumococcal genes associated with virulence bioinformatically, and demonstration of their presence in L. garvieae genome, for the first time suggest that they could be considered as potential virulence genes in this bacterium. Outcomes of the study provide a suitable resource for new projects, for analyzing the expression profile of $L$. garvieae isolates. In the long-term, the determination of prevalence of $L$. garvieae virulence genes will contribute to identifying the appropriate genomic targets in the development of new therapeutics in the fighting against the lactococcosis. 


\section{Acknowledgments}

The authors are grateful to Dr. Rebecca Wattam for providing valuable information about usage of PATRIC database. This research was financially supported by the Scientific Research Projects Coordination Unit of Istanbul University [project ID: 23352].

\section{References}

Casadevall, A., \& Pirofski, L.A. (1999). Host-pathogen interactions: redefining the basic concepts of virulence and pathogenicity. Infection and Immunity, 67 (8), 37033713. PMCid: PMC96643

Casadevall, A., \& Pirofski, L.A. (2000). Host-pathogen interactions: basic concepts of microbial commensalism, colonization, infection, and disease. Infection and Immunity, 68 (12), 6511-6518. PMCid: PMC97744

Castro, R., Reguera-Brito, M., López-Campos, G.H., Blanco, M.M., Aguado-Urda, M., Fernández-Garayzábal, J.F., \& Gibello, A. (2017). How does temperature influences the development of lactococcosis? Transcriptomic and immunoproteomic in vitro approaches. Journal of Fish Diseases, 40 (10), 1285-1297. https://doi.org/ $10.1111 / \mathrm{jfd} .12601$

Chhatwal, G.S. (2002). Anchorless adhesins and invasins of Gram-positive bacteria: a new class of virulence factors. Trends in Microbiology, 10 (5), 205-208. https://doi.org/10.1016/S0966-842X(02)02351-X

Desvaux, M., Dumas, E., Chafsey, I., \& Hebraud, M. (2006). Protein cell surface display in Gram-positive bacteria: from single protein to macromolecular protein structure. FEMS Microbiology Letters, 256 (1), 1-15. https://doi.org/10.1111/j.1574-6968.2006.00122.x

Gibello, A., Galán-Sánchez, F., Blanco, M. M., RodríguezIglesias, M., Domínguez, L., \& Fernández-Garayzábal, J. F. (2016). The zoonotic potential of Lactococcus garvieae: An overview on microbiology, epidemiology, virulence factors and relationship with its presence in foods. Research in Veterinary Science, 109, 59-70. https://doi.org/10.1016/j.rvsc.2016.09.010

Gillespie, J.J., Wattam, A.R., Cammer, S.A., Gabbard, J.L., Shukla, M.P., Dalay, O., ... Sobral, B.W. (2011). PATRIC: the comprehensive bacterial bioinformatics resource with a focus on human pathogenic species. Infection and Immunity, 79 (11), 4286-4298. https://doi.org/10.1128/IAl.00207-11

Hava, D.L., \& Camilli, A. (2002). Large-scale identification of serotype 4 Streptococcus pneumoniae virulence factors. Molecular Microbiology, 45 (5), 1389-1406. https://doi.org/10.1046/j.1365-2958.2002.03106.x

Kleerebezem, M., Hols, P., Bernard, E., Rolain, T., Zhou, M., Siezen, R. J., \& Bron, P. A. (2010). The extracellular biology of the lactobacilli. FEMS Microbiology Reviews. 34 (2), 199-230. https://doi.org/10.1111/j.15746976.2009.00208.x
Lau, G.W., Haataja, S., Lonetto, M., Kensit, S.E., Marra, A., Bryant, A.P., ... Holden, D.W. (2001). A functional genomic analysis of type 3 Streptococcus pneumoniae virulence. Molecular Microbiology, 40 (3), 555-571. https://doi.org/10.1046/j.1365-2958.2001.02335.x

Maniatis, T., Fritch, E.F., \& Sambrook, J. (2003). Molecular cloning: a laboratuary manual. NewYork, USA, Cold Spring Harbor Laboratory Press., 1448 pp.

Miyauchi, E., Toh, H., Nakano, A., Tanabe, S., \& Morita, H. (2012). Comparative genomic analysis of Lactococcus garvieae strains isolated from different sources reveals candidate virulence genes. International Journal of Microbiology, 2012. https://doi.org/10.1155/2012/728276

Morita, H., Toh, H., Oshima, K., Yoshizaki, M., Kawanishi, M., Nakaya, ... Hattori, M. (2011). Complete genome sequence and comparative analysis of the fish pathogen Lactococcus garvieae. PLoS One, 6 (8), e23184. https://doi.org/10.1371/journal.pone.0023184

NCBI, (2020). Lactococcus garvieae. Retrieved from https://www.ncbi.nlm.nih.gov/genome/genomes/699

Tandel, K., Bhatt, P., Ranjan, P., \& Rathi, K. R. (2017). Meningitis caused by Lactococcus garvieae. Medical Journal Armed Forces India, 73 (1), 94-96. https://doi.org/10.1016/j.mjafi.2015.08.004

Teker, T., Albayrak, G., Akayli, T., \& Urku, C. (2019) Detection of haemolysin genes as genetic determinants of virulence in Lactococcus garvieae. Turkish Journal of Fisheries and Aquatic Sciences, 19 (7), 625-634. https://doi.org/10.4194/1303-2712-v19_7_09

Ture, M., \& Altinok, I. (2016). Detection of putative virulence genes of Lactococcus garvieae. Diseases of Aquatic Organisms, 119 (1), 59-66. https://doi.org/10.3354/dao02981.

Vendrell D., Balca'zar J. L., Ruiz-Zarzuela I., de Blas I., Girone's O. \& Múzquiz, J. L. (2006). Lactococcus garvieae in fish: a review. Comparative Immunology, Microbiology and Infectious Diseases. 29 (4), 177-198. https://doi.org/10.1016/j.cimid.2006.06.003

Wang, C.Y.C., Shie, H.S., Chen, S.C., Huang, J.P., Hsieh, I.C., Wen, M.S., ... Wu, D. (2007). Lactococcus garvieae infections in humans: possible association with aquaculture outbreaks. International Journal of Clinical Practice, 61 (1), 68-73. https://doi.org/10.1111/j.17421241.2006.00855.x

Wattam, A.R., Abraham, D., Dalay, O., Disz, T.L., Driscoll, T., Gabbard, J.L., ... Sobral, B.W. (2014). PATRIC, the bacterial bioinformatics database and analysis resource. Nucleic Acids Research, 42 (D1), 581-591. https://doi.org/10.1093/nar/gkt1099

Zecconi, A., Cesaris, L., Liandris, E., Dapra, V., \& Piccinini, R. (2006). Role of several Staphylococcus aureus virulence factors on the inflammatory response in bovine mammary gland. Microbial Pathogenesis, 40 (4), 177183. https://doi.org/ 10.1016/j.micpath.2006.01.001 